\title{
Thermodynamic signature of a magnetic-field-driven phase transition within the superconducting state of an underdoped cuprate
}

\author{
J. B. Kemper ${ }^{1 \star}$, O. Vafek ${ }^{1}$, J. B. Betts ${ }^{2}$, F. F. Balakirev ${ }^{2}$, W. N. Hardy ${ }^{3,4}$, Ruixing Liang ${ }^{3,4}$, D. A. Bonn ${ }^{3,4}$ \\ and G. S. Boebinger ${ }^{1}$
}

More than a quarter century after the discovery of the high-temperature superconductor (HTS) $\mathrm{YBa}_{2} \mathrm{Cu}_{3} \mathrm{O}_{6+\delta}$ (YBCO; ref. 1), studies continue to uncover complexity in its phase diagram. In addition to HTS and the pseudogap ${ }^{2,3}$, there is growing evidence for multiple phases with boundaries which are functions of temperature $(T)$, doping $(p)$ and magnetic field $^{4-8}$. Here we report the low-temperature electronic specific heat $\left(C_{\text {elec }}\right)$ of $\mathrm{YBa}_{2} \mathrm{Cu}_{3} \mathrm{O}_{6.43}$ and $\mathrm{YBa}_{2} \mathrm{Cu}_{3} \mathrm{O}_{6.47}(p=0.076$ and $0.084)$ up to a magnetic field $(H)$ of $34.5 \mathrm{~T}$, a poorly understood region of the underdoped $H-T-p$ phase space. We observe two regimes in the low-temperature limit: below a characteristic magnetic field $H^{\prime} \approx 12-15 \mathrm{~T}, C_{\text {elec }} / T$ obeys an expected $H^{1 / 2}$ behaviour ${ }^{9,10}$; however, near $H^{\prime}$ there is a sharp inflection followed by a linear-in- $H$ behaviour. $H^{\prime}$ rests deep within the superconducting phase and, thus, the linear-in-H behaviour is observed in the zero-resistance regime"1. In the limit of zero temperature, $C_{\text {elec }} / T$ is proportional to the zero-energy electronic density of states. At one of our dopings, the inflection is sharp only at lowest temperatures, and we thus conclude that this inflection is evidence of a magnetic-field-driven quantum phase transition.

In elemental metals, the total low-temperature specific heat has the well-known form $C=\gamma T+\beta T^{3}-$ a sum of a linear term due to the electrons $\left(C_{\text {elec }}\right)$ and a cubic term due to the phonons. For ideal, perfectly clean $d_{x^{2}-y^{2}}$ superconductors, this form must be modified to $C(H=0, T)=\alpha T^{2}+\beta T^{3}$ owing to the linear electronic density of states that arises from the presence of symmetry-enforced nodal lines along which the momentumdependent superconducting gap, $\Delta_{\mathrm{k}}$, vanishes linearly with slope $\mathrm{d} \Delta_{\mathrm{k}} / \mathrm{d} k=v_{\Delta}$ (ref. 12). Experimental measurements of the lowtemperature specific heat of HTS cuprates in magnetic fields applied along the $c$-axis have found that $C_{\text {elec }}(H, T)=\gamma(H) T$ (refs 13,14), consistent with theoretically predicted scaling for $d$-wave quasiparticles experiencing an 'orbital' Doppler shift from superconducting vortices: $\gamma(H)-\gamma(H=0) \sim H^{1 / 2} / v_{\Delta}$ (ref. 9).

We have measured the specific heat of samples of $\mathrm{YBa}_{2} \mathrm{Cu}_{3} \mathrm{O}_{6.47}$ (abbreviated according to the convention YBCO6.47) with $T_{\mathrm{c}}=49 \mathrm{~K}$ and YBCO6.43 with $T_{\mathrm{c}}=41 \mathrm{~K}$, corresponding to hole dopings $p=0.084$ and 0.076 , respectively ${ }^{15}$. The total specific heat $(C)$ for $H<H^{\prime}$ strongly resembles previously reported results for YBCO6.56 (ref. 16). That is, for YBCO6.47, between
$1 \mathrm{~K}$ and $8 \mathrm{~K}$, we find $C_{\text {elec }}\left(H<H^{\prime}\right) / T=\gamma(H=0)+A_{c} H^{1 / 2}$, with $H^{\prime}=12 \mathrm{~T}, \gamma(H=0)=2.1 \mathrm{~mJ} \mathrm{~mol}^{-1} \mathrm{~K}^{-2}$ (Fig. 1) and $A_{\mathrm{c}}=0.6 \mathrm{~mJ} \mathrm{~mol}^{-1} \mathrm{~K}^{-2} \mathrm{~T}^{-1 / 2} \quad$ (Fig. 2). The phonon term, $\beta=0.38 \mathrm{~mJ} \mathrm{~mol}^{-1} \mathrm{~K}^{-4}$, varies negligibly $(<1 \%)$ over the full range of $H$ (Fig. 1). Our second doping, YBCO6.43, also approximately follows $C_{\text {elec }}\left(H<H^{\prime}\right) / T=\gamma(H=0)+A_{c} H^{1 / 2}$ below $3 \mathrm{~K}$, with $H^{\prime}=12 \mathrm{~T}, \gamma(H=0)=2.5 \mathrm{~mJ} \mathrm{~mol}^{-1} \mathrm{~K}^{-2}$ (Fig. 1) and $A_{\mathrm{c}}=0.6 \mathrm{~mJ} \mathrm{~mol}^{-1} \mathrm{~K}^{-2} \mathrm{~T}^{-1 / 2}$ (Fig. 2). The data from YBCO6.43 in Figs $1 \mathrm{~d}$ and $2 \mathrm{~b}$ have a greater dependence on temperature than the data for YBCO6.47 in Figs $1 b$ and $2 a$, yet they still follow the more general scaling law for $d$-wave superconductivity (ref. 10) below $H^{\prime} \approx 15 \mathrm{~T}$ (Fig. 3). For both YBCO6.43 and YBCO6.47, the values of $A_{c}$ are within experimental error of the value measured for YBCO6.56 of $A_{\mathrm{c}}=0.57 \mathrm{~mJ} \mathrm{~mol}^{-1} \mathrm{~K}^{-2} \mathrm{~T}^{-1 / 2}$ (ref. 16; see Supplementary Table 1 and Supplementary Fig. 8). At a further intermediate doping of $p=0.097$, YBCO6.51, we have measured similar samples up to $15 \mathrm{~T}$ and also find $\gamma(H=0)=2.3 \mathrm{~mJ} \mathrm{~mol}^{-1} \mathrm{~K}^{-2}$ and $A_{\mathrm{c}}=0.64 \mathrm{~mJ} \mathrm{~mol}^{-1} \mathrm{~K}^{-2} \mathrm{~T}^{-1 / 2}$ (see Supplementary Table 1 and Supplementary Figs 9 and 10). We infer from these $A_{\mathrm{c}}$ values that $v_{\Delta} \approx 0.15-0.17 \mathrm{eV} \AA$ (refs 17,18 ) and is insensitive to doping, despite the fact that the onset of finite resistance occurs at a magnetic field $\left(H_{\mathrm{R}}\right)$ that is a factor of $\sim 1.5$ higher in YBCO6.43 and YBCO6.47 than YBCO6.56 at low temperatures ${ }^{11}$ (see Supplementary Information). The value of $v_{\Delta}$ in YBCO for $p=0.08-0.10$ is strikingly similar to two other materials at the same dopings: in $\mathrm{Bi}_{2} \mathrm{Sr}_{2} \mathrm{CaCu}_{2} \mathrm{O}_{8+\delta}$ (BSCCO), as shown by scanning tunnelling microscopy (STM; ref. 19) and angle-resolved photoemission spectroscopy (ARPES) $\quad\left(v_{\Delta} \approx 0.11-0.12 \mathrm{eV} \AA\right.$; ref. 20), as well as values of $A_{c}$ from the specific heat of $\mathrm{La}_{2-x} \mathrm{Sr}_{x} \mathrm{CuO}_{4}$ (ref. 21), from which we determine $v_{\Delta} \approx 0.16 \mathrm{eV} \AA$ (refs 17,18). This constant magnitude of $v_{\Delta}$ for underdoped YBCO, with $T_{c}$ ranging from $41 \mathrm{~K}$ to $59 \mathrm{~K}$, which is similar to two other cuprates with $T_{c}$ varying from $20 \mathrm{~K}$ (underdoped LSCO; refs 17,18) to $92 \mathrm{~K}$ (near-optimum-doped BSCCO; ref. 20), strongly implies that the pairing scale (inferred from $v_{\Delta}$ ) does not determine $T_{c}$ for underdoped cuprates. $v_{\Delta}$ is relatively constant over the range of dopings recently studied by means of the specific heat ( $p=0.075-0.1$, see Supplementary Table 1$)$ and yet roughly a factor of two larger than $v_{\Delta}$ measured at optimal doping, despite $T_{c}$ in the latter being greater than $90 \mathrm{~K}$. That is, the energy gap is larger even though $T_{c}$ is lower in the underdoped samples studied here.

${ }^{1}$ Department of Physics and National High Magnetic Field Laboratory, Florida State University, Tallahassee, Florida 32310, USA. ${ }^{2}$ National High Magnetic Field Laboratory, Los Alamos National Laboratory, Los Alamos, New Mexico 87545, USA. ${ }^{3}$ Department of Physics and Astronomy, University of British Columbia, Vancouver, British Columbia V6T 1Z4, Canada. ${ }^{4}$ Canadian Institute for Advanced Research, 180 Dundas Street West, Suite 1400, Toronto, Ontario M5G 1Z8, Canada. *e-mail: jonathon.kemper@gmail.com 

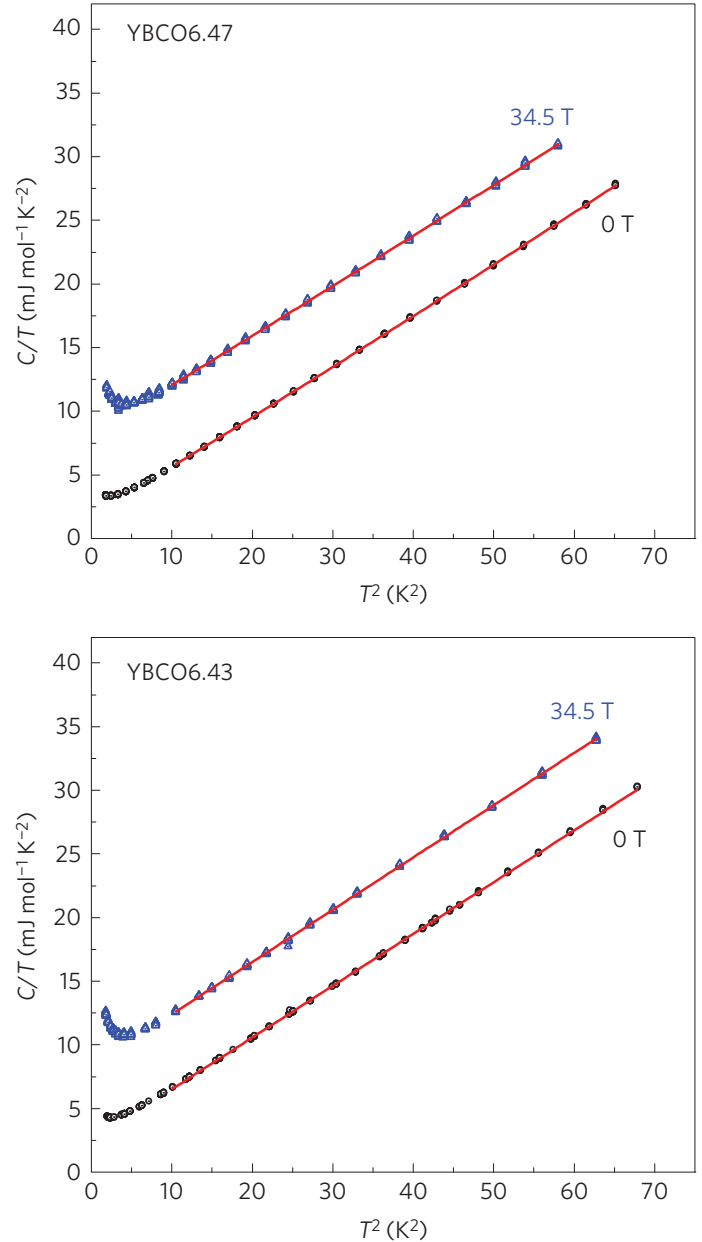

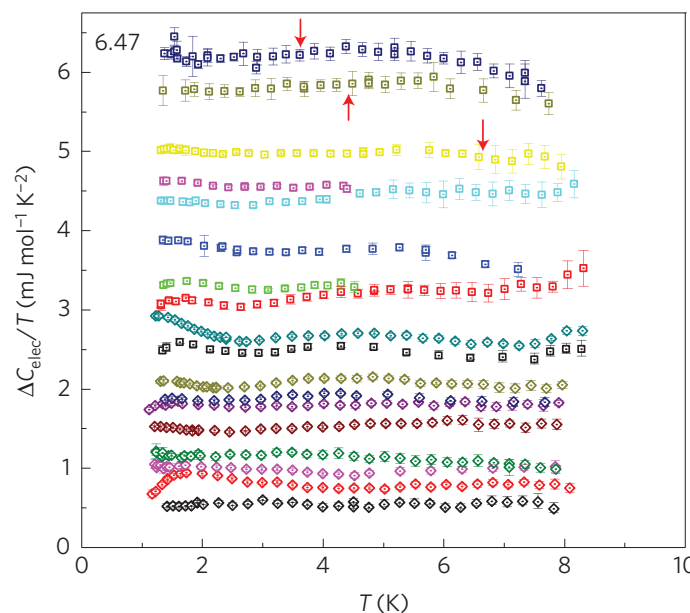

d

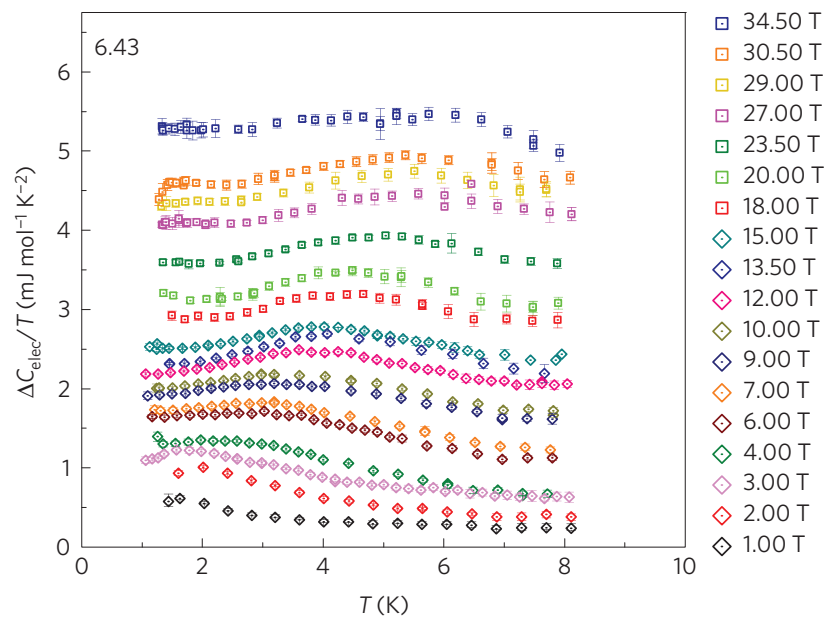

Figure 1 | Temperature dependence of the specific heat. $\mathbf{a}$, Total $C / T$ versus $T^{2}$ for YBCO6.47 at $H=0 \mathrm{~T}$ (black) and $34.5 \mathrm{~T}$ (blue) with linear fits (red) to determine $\gamma(H=0)=2.2 \mathrm{~mJ} \mathrm{~mol}^{-1} \mathrm{~K}^{-2}, \beta(H=0)=0.381 \mathrm{~mJ} \mathrm{~mol}^{-1} \mathrm{~K}^{-4}$ and $\beta(H=34.5 \mathrm{~T})=0.382 \mathrm{~mJ} \mathrm{~mol}^{-1} \mathrm{~K}^{-4}$. b, $\Delta C_{\text {elec }} / T$ versus $T$ for $Y B C O 6.47$ at various fields. c, As in a, but for YBCO6.43, giving $\gamma(H=0)=2.5 \mathrm{~mJ} \mathrm{~mol}^{-1} \mathrm{~K}^{-2}, \beta(H=0)=0.407 \mathrm{~mJ} \mathrm{~mol}^{-1} \mathrm{~K}^{-4}$ and $\beta(H=34.5 \mathrm{~T})=0.411 \mathrm{~mJ} \mathrm{~mol}^{-1} \mathrm{~K}^{-4}$ d, As in $\mathbf{b}$, but for YBCO6.43. Error bars in plots are \pm one standard deviation for a single data collection (see Supplementary Methods). The arrows in $\mathbf{b}$ indicate the approximate position of the resistive transition for $\mathrm{H} \geq 29 \mathrm{~T}$ as reported for YBCO6.47 (ref. 11).

We now note that the predicted $H=0$ superconducting term $C \sim \alpha T^{2}$ is not clearly evident; however, we can conclude $\alpha<0.2 \mathrm{~mJ} \mathrm{~mol}^{-1} \mathrm{~K}^{-3}$ (see Supplementary Methods). We note further that other specific heat ${ }^{13,14}$ and ARPES (ref. 22) measurements are consistent with this upper bound, given that $\alpha=18 \zeta(3) k_{\mathrm{B}}^{3} n_{l} a b\left(1 / \pi \hbar^{2} v_{\mathrm{F}} v_{\Delta}\right)$ (ref. 12), where $v_{\mathrm{F}}$ is the Fermi velocity, $\zeta(x)$ is the Riemann zeta function, $a b$ is the area of the $a-b$ plane per unit cell, and $n_{l}=2$ is the number of $\mathrm{CuO}_{2}$ layers per mole (see Supplementary Information).

The most striking feature uncovered by the present study is the obvious deviation from $\Delta C_{\text {elec }}=C_{\text {elec }}(H, T)-C_{\text {elec }}(H=0, T) \sim H^{1 / 2}$ (see Supplementary Methods) characterized by a low-temperature inflection point and subsequent $\Delta C_{\text {elec }} \sim H$ behaviour, as seen in Fig. 2. This approximately linear-in- $H$ behaviour, unprecedented for HTS cuprates, leads to $\Delta C_{\text {elec }}(H=34.5 \mathrm{~T}) / T \approx 5-6 \mathrm{~mJ} \mathrm{~mol}^{-1} \mathrm{~K}^{-2}$ (Fig. 1b), roughly twice the value observed in YBCO6.56 at any $H \leq 45 \mathrm{~T}$ (ref. 16). Two key facts are evident: (1) a larger specific heat exists at the lower dopings, YBCO6.43 and YBCO6.47, than at the higher doping, YBCO6.56. This is perhaps counterintuitive because superconductivity is more robust at the lower dopings, thus one might expect a larger superconducting gap, which would ordinarily lead to a lower specific heat. That is, at $T \approx 1 \mathrm{~K}, H_{\mathrm{R}} \approx 45 \mathrm{~T}$ for YBCO6.43 and YBCO6.47, whereas $H_{\mathrm{R}}<35 \mathrm{~T}$ for YBCO6.56 (refs 11,16; see Supplementary Information). (2) In YBCO6.56, over any portion of the magnetic field range measured, all evidence points to the absence of linear-in- $H$ enhancement of $C_{\text {elec }}$ above the fit extrapolated from low-field data ${ }^{16}$. Moreover, $\Delta C_{\text {elec }} / T$ is essentially independent of temperature in YBCO6.47, even for data crossing into the resistive regime at $H_{\mathrm{R}}(T)$, indicated by the arrows in Fig. $1 \mathrm{~b}$ (ref. 11). The magnetic field dependence of $\Delta C_{\text {elec }} / T$ is also not affected by $H_{\mathrm{R}}(T)$. These facts, taken together, make it unlikely that $H^{\prime}$ and $H_{\mathrm{R}}(T)$ mark the mean-field suppression of the superconducting gap by a magnetic field. In addition, the abruptness of the change at $H^{\prime}$ is at odds with the smoothness expected in the magnetic field suppression of the order parameter of a $d$-wave superconductor ${ }^{23}$.

In Fig. 3, we re-plot the YBCO6.43 data to test for a more general $d$-wave scaling (SL scaling) predicted by Simon and Lee ${ }^{10}$. All data below $H^{\prime}=15 \mathrm{~T}$ scale within the scatter between 1 and $7 \mathrm{~K}$. The breakdown in scaling above $15 \mathrm{~T}$ is most clearly visible in the marked upturn in the $2.5 \mathrm{~K}$ trace (Fig. 3), which represents a sharp deviation from scaling. Note that all data for $H \geq 18 \mathrm{~T}$ are above $H^{\prime}$, and do not scale. A broad maximum centred near $1 \mathrm{~K} \mathrm{~T}^{-1 / 2}$ exists for all fields. The quotient $T / H^{1 / 2}$ is proportional to the ratio of the orbital magnetic length over the thermal length, suggesting the maximum may arise from superconducting vortices: in fact, a similar anomaly in the low-temperature $C_{\text {elec }}$ of $d$-wave superconductors has been predicted as a consequence of magnetic sub-bands resulting from the periodicity of the vortex lattice ${ }^{24}$. 
a

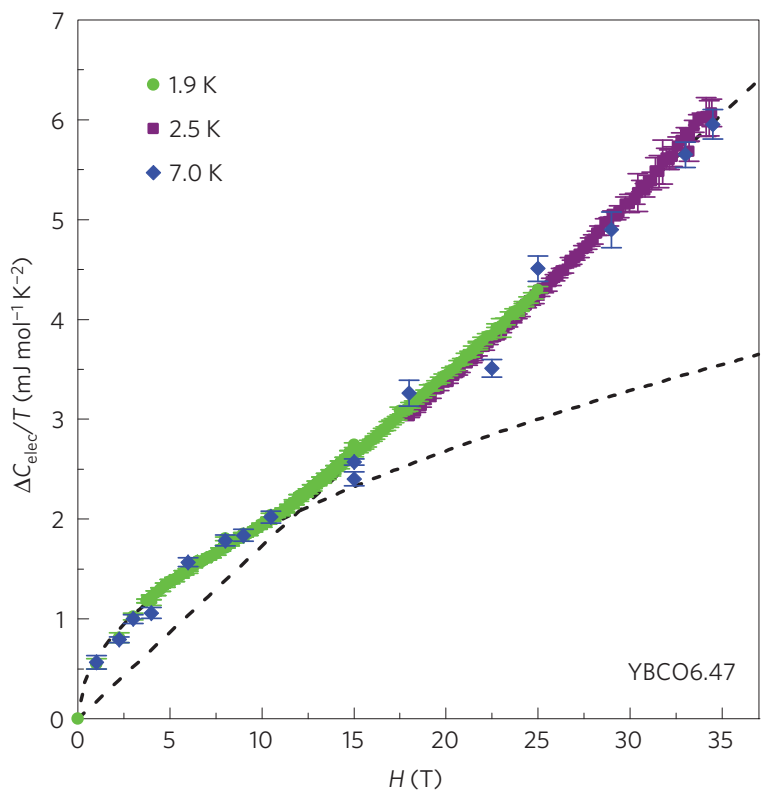

b

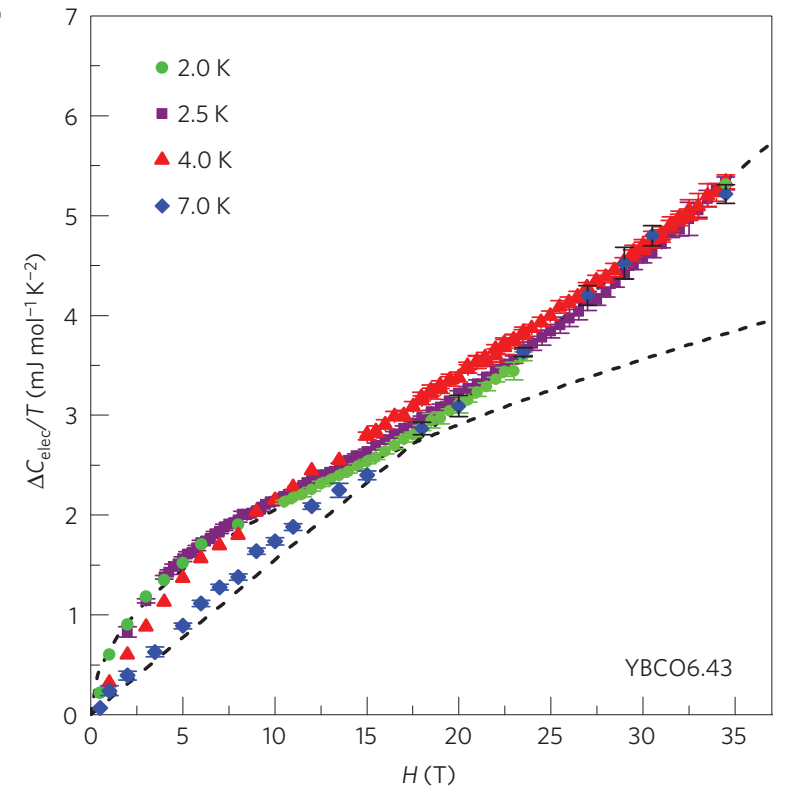

Figure 2 | Field dependence of electronic specific heat. $\mathbf{a}, \Delta C_{\text {elec }} / T$ versus $H$ for $Y B C O 6.47$. The dashed black curve and line are guides to the eye, given by $\Delta C_{\text {elec }} / T=0.6 \mathrm{~mJ} \mathrm{~mol}^{-1} \mathrm{~K}^{-2} \mathrm{~T}^{-1 / 2} \times H^{1 / 2}$ and $\Delta C_{\text {elec }} / T=0.17 \mathrm{~mJ} \mathrm{~mol}{ }^{-1} \mathrm{~K}^{-2} \mathrm{~T}^{-1} \times H$, respectively, where $H$ is given in tesla. $\mathbf{b}, \Delta C_{\text {elec }} / T$ versus $H$ for YBCO6.43. Again, the dashed curve and line are guides to the eye: $\Delta C_{\text {elec }} / T=0.6 \mathrm{~mJ} \mathrm{~mol}^{-1} \mathrm{~K}^{-2} \mathrm{~T}^{-1 / 2} \times H^{1 / 2}$ and $\Delta C_{\text {elec }} / T=0.155 \mathrm{~mJ} \mathrm{~mol}^{-1} \mathrm{~K}^{-2} \mathrm{~T}^{-1} \times H_{\text {, }}$ respectively. Error bars in plots are \pm one standard deviation for a single data collection (see Supplementary Methods).

We re-plot the $\sim 2 \mathrm{~K}$ data of Fig. 2a in Fig. 4a to demonstrate the breakdown of orbital scaling at $H^{\prime}$, and to clearly illustrate the sharpness of the transition between the two regimes. The plot depicts a scenario in which the orbital effect, $\Delta C_{\text {elec }}(H) / T \propto H^{1 / 2} / v_{\Delta}$, is responsible for the observed $\Delta C_{\text {elec }}(H) / T$ over the entire magnetic field range. Such a scenario would necessitate a field-dependent $v_{\Delta}{ }^{\text {eff }}(H) \propto T H^{1 / 2} / \Delta C_{\text {elec }}(H)$ (where $v_{\Delta}{ }^{\text {eff }}$ is an effective field-dependent parameter such that, for $H \rightarrow 0, v_{\Delta}{ }^{\text {eff }}=v_{\Delta}$ ) that is more or less a piecewise function: $v_{\Delta}{ }^{\text {eff }}$ is largely independent of field below $H^{\prime}$, followed by a sudden drop at $H^{\prime}$ that asymptotes to $v_{\Delta}{ }^{\text {eff }} \propto 1 / H^{1 / 2}$ (Fig. 4a). As $v_{\Delta}{ }^{\text {eff }}$ is proportional to the magnitude of the superconducting order parameter, this further disfavours a mean-field scenario involving suppression of the gap by $H$ (ref. 23).

Instead, it is natural to think of Zeeman splitting giving rise to linear-in- $H$ specific heat, which can result from Zeeman splitting of $d$-wave quasiparticles at sufficiently high fields ${ }^{25}$. In such a case, the high-field slope $\Delta C_{\text {elec }}(H) / T H \sim\left(v_{\mathrm{F}} v_{\Delta}\right)^{-1}$, and-assuming a $g$-factor of 2 -our value for this slope, $\approx 0.16 \mathrm{~mJ} \mathrm{~mol}^{-1} \mathrm{~K}^{-2} \mathrm{~T}^{-1}$, determines $\alpha \approx 0.37 \mathrm{~mJ} \mathrm{~mol}^{-1} \mathrm{~K}^{-3}$ (refs 12,25 ). This violates our established zero-field bound on $\alpha$ by almost a factor of two, and requires a marked drop in the product $v_{\mathrm{F}}{ }^{\text {eff }} v_{\Delta}{ }^{\text {eff }}$ over a small field range around $H^{\prime}$ for this Zeeman scenario to be internally consistent.

Figure $4 \mathrm{~b}$ presents this second scenario, with fixed $v_{\Delta}$ (determined from $A_{\mathfrak{c}}$, the prefactor of the square root $H$ ) and a field-dependent $v_{\mathrm{F}}$ eff calculated to fit the low-temperature data. The magnitude of $v_{\mathrm{F}}{ }_{\mathrm{F}}^{\text {fff }}$ decreases by at least a factor of three from its low-field value, suggesting the sudden onset of a mass enhancement at $H^{\prime}$. The phase diagram of YBCO (Fig. 5) supports this picture and provides a scenario for understanding the observed phase transition: in the resistive state, using pulsed magnetic fields above $50 \mathrm{~T}$, quantum oscillations (QO; ref. 26) and resistivity measurements $\mathrm{s}^{27}$ have been interpreted in terms of a metal-to-insulator transition (MIT) near YBCO6.47, with a divergent cyclotron mass, $m^{*}$ (ref. 26). In light of Fig. $4 \mathrm{~b}$, we propose that this enhanced mass is a field-sensitive phenomenon, which increases rapidly at $H^{\prime}$ and drives the system into a
Zeeman-dominated regime. We note that $v_{\mathrm{F}}$ eff $/ v_{\Delta} \approx 2 / 3$ above $20 \mathrm{~T}$ (see Supplementary Information), a violation of the requirement that $v_{\mathrm{F}} \gg v_{\Delta}$ in the orbital-scaling regime, providing a further quantitative indication that the high-field regime is physically distinct from the low-field regime.

A proposed quantum critical point at $p=0.08$ (refs 27,28), linked to the high-field mass enhancement ${ }^{26}$, has been associated in YBCO with the boundary of a spin density wave (SDW) phase seen by means of neutron diffraction and muon spin rotation ( $\mu$ SR; refs 5,6; Fig. 5), whose order is enhanced by magnetic field ${ }^{6}$. Our high-field state may be linked to this SDW and its associated critical point.

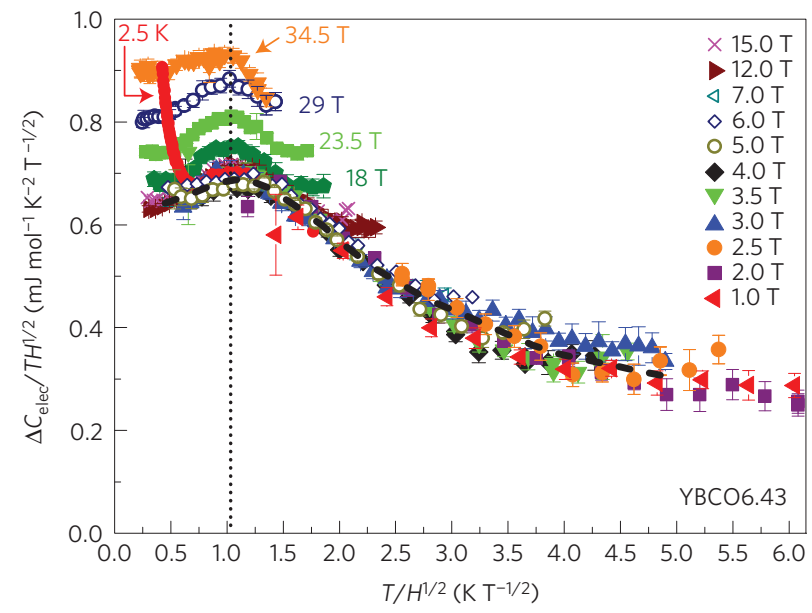

Figure $\mathbf{3}$ | Scaling plot. Data from Fig. 1d plus the $2.5 \mathrm{~K}$ data from Fig. $2 \mathrm{~b}$ (red circles) plotted to test for SL scaling for $d$-wave superconductivity (ref. 10). The data collapse within error for $T \leq 7 \mathrm{~K}$, provided $H \leq 15 \mathrm{~T}$. The dashed black curve traces an approximate scaling function. The legend lists fixed-field curves that follow scaling below $\approx 7 \mathrm{~K}$. The dotted vertical line indicates an anomaly consisting of a maximum at $T / H^{1 / 2} \approx 1 \mathrm{KT}^{-1 / 2}$ at all fields. The red arrow indicates the upturn in the $2.5 \mathrm{~K}$ data. Error bars in plots are \pm one standard deviation for a data collection. 


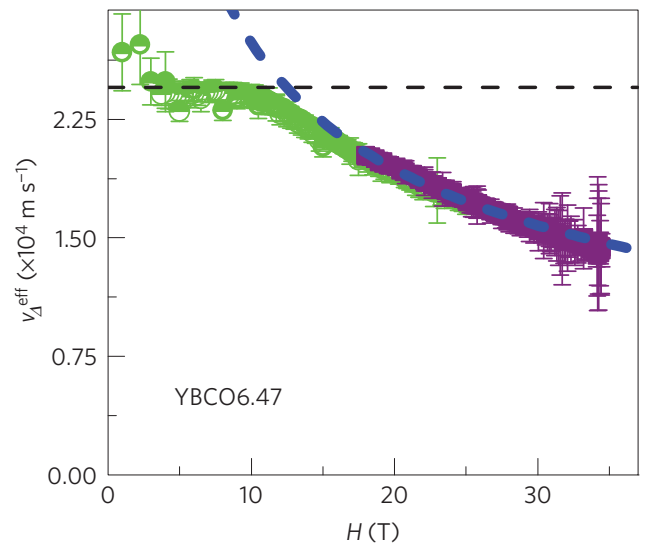

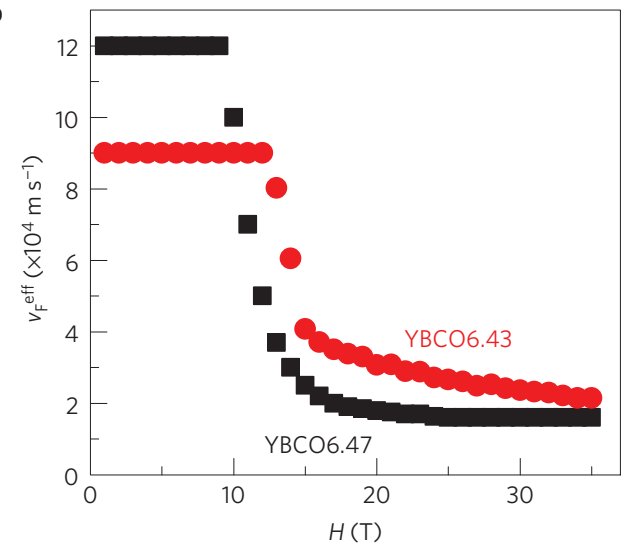

Figure 4 | Calculated effective electronic velocity parameters. a, Re-plotting of the data in Fig. 2a as $v_{\Delta}$ eff versus $H_{,}\left(v_{\Delta} \propto T H^{1 / 2} / \Delta C_{\text {elec }}\right.$ for ideal $d$-wave superconductors with constant $v_{F}{ }^{\text {eff }}$ ). The colour scheme is the same as in Fig. $2 \mathrm{a}$, and dashed lines are again defined by $\Delta C_{\text {elec }} / T=0.17 \mathrm{~mJ} \mathrm{~mol}^{-1} \mathrm{~K}^{-2} \mathrm{~T}^{-1} \times \mathrm{H}_{\text {and }} \Delta C_{\text {elec }} / T=0.6 \mathrm{~mJ} \mathrm{~mol}^{-1} \mathrm{~K}^{-2} \mathrm{~T}^{-1 / 2} \times \mathrm{H}^{1 / 2}$. Error bars represent \pm one standard deviation for one data collection, recalculated for the quantity $\mathrm{TH}^{1 / 2} / \Delta C_{\text {elec }}$. $\mathbf{b}$, Alternative scenario assuming fixed $v_{\Delta}$, showing calculated $v_{F}{ }^{\text {eff }}$ (ref. 18) with both orbital and Zeeman effects included. The value of $v_{\mathrm{F}}{ }^{\text {eff }}$ below $H^{\prime}$ is determined from the thermal conductivity ${ }^{30}$. The drop in $v_{F}{ }^{\text {eff }}$ implies an enhanced quasiparticle mass above $H^{\prime}$.

Indeed, a field-driven transition to a SDW state-coexisting with SC-has been reported in the cuprates, although this observation was in a different material $\left(\mathrm{La}_{2-x} \mathrm{Sr}_{x} \mathrm{CuO}_{4}\right)$ at a much higher doping $(p=0.14$; ref. 29). Unfortunately, the most comprehensive survey of the specific heat of $\mathrm{La}_{2-x} \mathrm{Sr}_{x} \mathrm{CuO}_{4}$ (ref. 21) does not include a sample at a doping that would traverse this reported phase boundary. A neutron scattering or nuclear magnetic resonance (NMR) experiment in YBCO at these dopings and magnetic fields might provide further evidence of SDW order underlying the magnetic-field-driven phase transition that we report here.

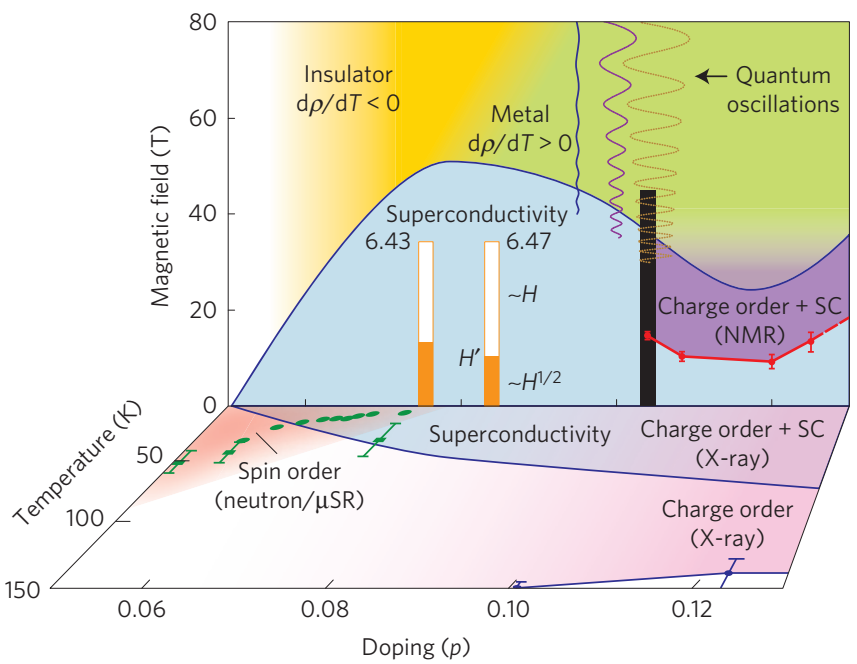

Figure $\mathbf{5}$ | Phase diagram of YBCO. The sections represent the $H$-p plane for $T \rightarrow 0$ and the $T-p$ plane for $H \rightarrow 0$. Orange/white rectangles represent the range of the present measurements, and the black rectangle represents ref. 16. Orange and white represent the range of $d$-wave scaling (that is, $C_{\text {elec }} / T \sim H^{1 / 2}$ ) and linear-in- $H$ behaviour, respectively. $H^{\prime}$ marks the phase transition. Superconductivity (SC) phase boundaries (blue curves) are from refs 11,15. Metal-to-insulator (MIT) boundary (green/yellow) was determined from resistivity ${ }^{27}$ and QO (ref. 26). Charge density wave (CDW) was established via NMR (ref. 7) (purple, red circle/line) and $\mathrm{X}$-rays 8,30 (pink, blue circle/line, CDW may extend below $p=0.08$ (ref. 28)). Spin density wave (red) at $H=0$, as determined by neutron scattering ${ }^{6}$ (green squares) and $\mu S R$ (refs 4,5 ) (green circles), vanishes near $p=0.08$
Received 14 August 2014; accepted 9 September 2015; published online 19 October 2015

\section{References}

1. Wu, M. K. et al. Superconductivity at $93 \mathrm{~K}$ in a new mixed-phase $\mathrm{Y}-\mathrm{Ba}-\mathrm{Cu}-\mathrm{O}$ compound system at ambient pressure. Phys. Rev. Lett. 58, 908-910 (1987).

2. Alloul, H., Ohno, T. \& Mendels, P. ${ }^{89} \mathrm{Y}$ NMR evidence for a Fermi-liquid behavior in $\mathrm{YBa}_{2} \mathrm{Cu}_{3} \mathrm{O}_{6+x}$. Phys. Rev. Lett. 63, 1700-1703 (1989).

3. Walstedt, R. E. \& Warren, W. W. Nuclear resonance properties of $\mathrm{YBa}_{2} \mathrm{Cu}_{3} \mathrm{O}_{6+x}$ superconductors. Science 248, 1082-1087 (1990).

4. Sonier, J. E. et al. Spin-glass state of individual magnetic vortices in $\mathrm{YBa}_{2} \mathrm{Cu}_{3} \mathrm{O}_{y}$ and $\mathrm{La}_{2-x} \mathrm{Sr}_{x} \mathrm{CuO}_{4}$ below the metal-to-insulator crossover. Phys. Rev. B 76, 064522 (2007).

5. Coneri, F., Sanna, S., Zheng, K., Lord, J. \& DeRenzi, R. Magnetic states of lightly hole-doped cuprates in the clean limit as seen via zero-field muon spin spectroscopy. Phys. Rev. B 81, 104507 (2010).

6. Haug, D. et al. Neutron scattering study of the magnetic phase diagram of underdoped $\mathrm{YBa}_{2} \mathrm{Cu}_{3} \mathrm{O}_{6+x}$. New J. Phys. 12, 105006 (2010).

7. Wu, T. et al. Emergence of charge order from the vortex state of a high-temperature superconductor. Nature Commun. 4, 2113 (2013).

8. Ghiringhelli, G. et al. Long-range incommensurate charge fluctuations in (Y,Nd) $\mathrm{Ba}_{2} \mathrm{Cu}_{3} \mathrm{O}_{6+x}$. Science 337, 821-825 (2012).

9. Volovik, G. Superconductivity with lines of gap nodes: Density of states in the vortex. JETP Lett. 58, 457-461 (1993).

10. Simon, S. H. \& Lee, P. A. Scaling of the quasiparticle spectrum for $d$-wave superconductors. Phys. Rev. Lett. 78, 1548-1551 (1997).

11. Ramshaw, B. J. et al. Vortex lattice melting and $H_{c 2}$ in underdoped $\mathrm{YBa}_{2} \mathrm{Cu}_{3} \mathrm{O}_{y}$. Phys. Rev. B 86, 174501 (2012).

12. Kopnin, N. \& Volovik, G. Singularity of the vortex density of states in $d$-wave superconductors. JETP Lett. 64, 690-694 (1996).

13. Moler, K. A. et al. Magnetic field dependence of the density of states of $\mathrm{YBa}_{2} \mathrm{Cu}_{3} \mathrm{O}_{6.95}$ as determined from the specific heat. Phys. Rev. Lett. 73, 2744-2747 (1994)

14. Wang, Y., Revaz, B., Erb, A. \& Junod, A. Direct observation and anisotropy of the contribution of gap nodes in the low-temperature specific heat of $\mathrm{YBa}_{2} \mathrm{Cu}_{3} \mathrm{O}_{7}$. Phys. Rev. B 63, 094508 (2001).

15. Liang, R., Bonn, D. A. \& Hardy, W. N. Evaluation of $\mathrm{CuO}_{2}$ plane hole doping in $\mathrm{YBa}_{2} \mathrm{Cu}_{3} \mathrm{O}_{6+x}$ single crystals. Phys. Rev. B. 73, 180505 (2006).

16. Riggs, S. C. et al. Heat capacity through the magnetic-field-induced resistive transition in an underdoped high-temperature superconductor. Nature Phys. 7, $332-335$ (2011)

17. Ioffe, L. \& Millis, A. $d$-wave superconductivity in doped Mott insulators. J. Phys. Chem. Solids 63, 2259-2268 (2002).

18. Cvetkovic, V. \& Vafek, O. Berry phases and the intrinsic thermal Hall effect in high-temperature cuprate superconductors. Nature Commun. 6, 6518 (2015).

19. Pushp, A. et al. Extending universal nodal excitations optimizes superconductivity in $\mathrm{Bi}_{2} \mathrm{Sr}_{2} \mathrm{CaCu}_{2} \mathrm{O}_{8+\delta}$. Science 324, 1689-1693 (2009).

20. Vishik, I. M. et al. Phase competition in trisected superconducting dome. Proc. Natl Acad. Sci. USA 109, 18332-18337 (2012). 
21. Wen, H. H. et al. Pseudogap, superconducting energy scale, and Fermi arcs of underdoped cuprate superconductors. Phys. Rev. B 72, 134507 (2005).

22. Vishik, I. M. et al. Doping-dependent nodal Fermi velocity of the high-temperature superconductor $\mathrm{Bi}_{2} \mathrm{Sr}_{2} \mathrm{CaCu}_{2} \mathrm{O}_{8+\delta}$ revealed using high-resolution angle-resolved photoemission spectroscopy. Phys. Rev. Lett 104, 207002 (2010)

23. Watanabe, K., Kita, T. \& Arai, M. Magnetic-field dependence of thermodynamic quantities in the vortex state of type-II superconductors. Phys. Rev. B 71, 144515 (2005).

24. Melikyan, A. \& Vafek, O. Quantum oscillations in the mixed state of $d$-wave superconductors. Phys. Rev. B 78, 020502 (2008).

25. Yang, K. \& Sondhi, S. L. Response of a $d_{x^{2}-y^{2}}$ superconductor to a Zeeman magnetic field. Phys. Rev. B 57, 8566-8570 (1998).

26. Sebastian, S. E. et al. Metal-insulator quantum critical point beneath the high $T_{c}$ superconducting dome. Proc. Natl Acad. Sci. USA 107, 6175-6179 (2010).

27. Leboeuf, D. et al. Lifshitz critical point in the cuprate superconductor $\mathrm{YBa}_{2} \mathrm{Cu}_{3} \mathrm{O}_{y}$ from high-field Hall effect measurements. Phys. Rev. B 83, 054506 (2011).

28. Hücker, M. et al. Competing charge, spin, and superconducting orders in underdoped $\mathrm{YBa}_{2} \mathrm{Cu}_{3} \mathrm{O}_{y}$. Phys. Rev. B 90, 054514 (2014).

29. Khaykovich, B. et al. Field-induced transition between magnetically disordered and ordered phases in underdoped $\mathrm{La}_{2-x} \mathrm{Sr}_{x} \mathrm{CuO}_{4}$. Phys. Rev. B 71, 220508 (2005).

30. Sutherland, M. et al. Thermal conductivity across the phase diagram of cuprates: Low-energy quasiparticles and doping dependence of the superconducting gap. Phys. Rev. B 67, 174520 (2003).

\section{Acknowledgements}

The authors thank S. Kivelson, R. Baumbach, A. Kapitulnik, M. Norman, B. Ramshaw, A. Shekhter, J. Sonier and S. Riggs for discussions and commentary on the manuscript, as well as A. Migliori for discussions on the experimental techniques. J.B.K. thanks C. Moir for assistance during experiments. A portion of this work was performed at the National High Magnetic Field Laboratory, which is supported by National Science Foundation Cooperative Agreement No. DMR-1157490, the State of Florida, and the US Department of Energy. Work at the University of British Columbia was supported by the Natural Science and Engineering Research Council of Canada and the Canadian Institute for Advanced Research.

\section{Author contributions}

W.N.H., R.L. and D.A.B. prepared the samples and contributed to the experimental plan. J.B.K. refined the experimental set-up, performed the experiments, analysed the data and contributed to the writing of the manuscript. O.V. contributed to the writing of the manuscript and interpretation of results. G.S.B. supervised the project and contributed to the writing of the manuscript. F.F.B. contributed to the experimental software and thermometer calibrations. J.B.B. contributed to the experimental hardware and thermometer calibrations. F.F.B., J.B.B. and D.A.B. provided comments on the results and manuscript.

\section{Additional information}

Supplementary information is available in the online version of the paper. Reprints and permissions information is available online at www.nature.com/reprints.

Correspondence and requests for materials should be addressed to J.B.K.

\section{Competing financial interests}

The authors declare no competing financial interests. 